\title{
Comparison of fundamental, second harmonic, and superharmonic imaging: A simulation study
}

\author{
Paul L. M. J. van Neer ${ }^{\text {a) }}$ and Mikhail G. Danilouchkine ${ }^{\text {b) }}$ \\ Department of Biomedical Engineering, Erasmus MC, P.O. Box 2040, 3000 CA, Rotterdam, The Netherlands \\ Martin D. Verweij
Laboratory of Electromagnetic Research, Faculty of Electrical Engineering, Mathematics and
Computer Science, Delft University of Technology, Mekelweg 4, 2628 CD, Delft, The Netherland
}

Libertario Demi

Lab of Acoustical Imaging and Sound Control, Faculty of Applied Sciences, Delft University of Technology, Lorentzweg 1, 2628 CJ, Delft, The Netherlands

Marco M. Voormolen,

Department of Circulation and Imaging (ISB), Norwegian University of Science and Technology (NTNU), Prinsesse Kristinas gate 3, Trondheim, Norway

Anton F. W. van der Steen ${ }^{\text {b) }}$ and Nico de Jong ${ }^{\text {c) }}$
Department of Biomedical Engineering, Erasmus MC, P.O. Box 2040, 3000 CA, Rotterdam, The Netherlands

(Received 20 February 2011; revised 27 August 2011; accepted 1 September 2011)

In medical ultrasound, fundamental imaging (FI) uses the reflected echoes from the same spectral band as that of the emitted pulse. The transmission frequency determines the trade-off between penetration depth and spatial resolution. Tissue harmonic imaging (THI) employs the second harmonic of the emitted frequency band to construct images. Recently, superharmonic imaging (SHI) has been introduced, which uses the third to the fifth (super) harmonics. The harmonic level is determined by two competing phenomena: nonlinear propagation and frequency dependent attenuation. Thus, the transmission frequency yielding the optimal trade-off between the spatial resolution and the penetration depth differs for THI and SHI. This paper quantitatively compares the concepts of fundamental, second harmonic, and superharmonic echocardiography at their optimal transmission frequencies. Forward propagation is modeled using a 3D-KZK implementation and the iterative nonlinear contrast source (INCS) method. Backpropagation is assumed to be linear. Results show that the fundamental lateral beamwidth is the narrowest at focus, while the superharmonic one is narrower outside the focus. The lateral superharmonic roll-off exceeds the fundamental and second harmonic roll-off. Also, the axial resolution of SHI exceeds that of FI and THI. The far-field pulseecho superharmonic pressure is lower than that of the fundamental and second harmonic. SHI appears suited for echocardiography and is expected to improve its image quality at the cost of a slight reduction in depth-of-field. (c) 2011 Acoustical Society of America. [DOI: 10.1121/1.3643815]

PACS number(s): 43.80.Qf, 43.80.Vj, 43.35.Yb, 43.35.Bf [CCC] Pages: 3148-3157

\section{INTRODUCTION}

Over the last decades, medical ultrasound has greatly improved due to numerous technological advances. ${ }^{1}$ However, until the late 1990s most of these improvements mainly impacted the technically suitable patient subgroup. ${ }^{1,2}$ A considerable subgroup of patients was considered difficult to image due to tough windows, inhomogeneous skin layers, and limited penetration. ${ }^{2}$ This was especially so in the case

\footnotetext{
${ }^{a)}$ Current address: Medical Technology Group, Department of Precision Motion Systems, TNO, P.O. Box 155, 2600 AD, Delft, the Netherlands.

b) Also at: The Interuniversity Cardiology Institute of the Netherlands, P. O. Box 19258, 3501 DG, Utrecht, the Netherlands.

c) Author to whom correspondence should be addressed. Also at: The Interuniversity Cardiology Institute of the Netherlands, P. O. Box 19258, 3501 DG, Utrecht, the Netherlands; and the Department of Physics of Fluids, University of Twente, P. O. Box 217, 7522 NB, Enschede, the Netherlands. Electronic mail: n.dejong@erasmusmc.nl
}

of echocardiography, where the ultrasound propagation paths are generally long and reflections from the skeletal structures occur often. In this early period ultrasound imaging was based on linear acoustic wave propagation. The technique was called fundamental imaging (FI) because the frequency transmitted (the fundamental frequency) was also received and used to construct an image. The transmission frequency follows from a trade-off between the attainable spatial resolution, which improves for increasing frequency, and the required penetration depth, which reduces for an increasing frequency. ${ }^{3}$ The trade-off yields an optimum frequency that depends on the specific application. For example, for the visualization of the left ventricular endocardial border during echocardiography (imaging depths of 10-15 cm) a transmission frequency of $3.5 \mathrm{MHz}$ yielded the best results. ${ }^{4}$

About a decade ago it became possible to improve ultrasound image quality by exploiting the nonlinear nature of acoustic wave propagation. The developed technique is called 
tissue second harmonic imaging (THI) and is based on the selective imaging of the reflections of the second harmonic of the emitted frequency. ${ }^{2,5}$ Compared to fundamental imaging, second harmonic imaging features lower sidelobes, and is therefore less sensitive to clutter and off-axis scatterers. ${ }^{1,3,6-8}$ Also, since the second harmonic field builds up progressively, the effects of reverberation and near-field artifacts are greatly reduced. ${ }^{1,8}$ This is particularly important for echocardiography in view of the proximity of the ribs to the available imaging windows, and the intermediate skin and fat layers. Ultrasound image quality improved considerably due to these characteristics, especially for the patient subgroup considered challenging to image. ${ }^{8}$

Even though the nonlinear nature of wave propagation in tissue was already used in the late 1970s for acoustic microscopy $^{9}$ and shown to be relevant in the context of medical applications in the early to mid 1980s by Muir and Carstensen ${ }^{10}$ and Starritt et al., ${ }^{11}$ it took until the late 1990s for second harmonic imaging to take off. Reasons for this were the necessary improvements in dynamic range and signal processing, but also the widely held assumption that nonlinear distortion was not a significant factor in medical diagnostic imaging, since the frequency dependent thermoviscous absorption rapidly dissipates the generated harmonic energy. ${ }^{2}$ The level of the harmonics is determined by two competing phenomena: A growth over distance of the harmonics due to nonlinear propagation, and a decay over distance of the harmonics due to frequency dependent attenuation. Therefore, compared to fundamental imaging, second harmonic imaging requires a different trade-off between the required penetration depth and the obtainable spatial resolution, and this will result in a different optimal transmission frequency. Thus, in the case of visualizing the left ventricular endocardial border during echocardiography (imaging depths of 10-15 cm) a transmission frequency of 1.6-1.8 MHz yielded the best results for second harmonic imaging. This in contrast to the $3.5 \mathrm{MHz}$ transmission frequency that gave the optimum results for fundamental imaging. ${ }^{4}$

Recently, a new imaging modality named tissue superharmonic imaging (SHI) was proposed. The modality extends the idea behind second harmonic imaging by using the reflections of the third to the fifth harmonics arising from nonlinear wave propagation. ${ }^{13}$ Based on numerical simulations, ${ }^{8}$ superharmonic imaging promises increased suppression of near-field artifacts, reverberations, and offaxis artifacts in addition to enhancing the spatial resolution. The resulting images from phantom measurements showed indeed more details than those produced by second harmonic imaging. ${ }^{8}$ Recently, this was confirmed in additional simulations and in vitro experiments conducted by Ma et al. ${ }^{14}$ As superharmonic imaging uses the third to the fifth harmonics for imaging instead of the second harmonic, a different transmission frequency is expected to yield the optimum trade-off between spatial resolution and penetration depth. For superharmonic echocardiography optimal transmission frequencies of $1.0-1.2 \mathrm{MHz}$ were found. ${ }^{12,15}$

Although the physical principles of the aforementioned imaging methods are different, there seems to exist a common property: For the same application, the ranges of the frequencies used to create an image are very similar. This is illustrated by the case of echocardiography, where the center frequencies of the imaging signals are $3.5 \mathrm{MHz}$ for fundamental imaging, 3.4-3.6 MHz for second harmonic imaging, and 3.0-6.0 (with 3.0-3.6 MHz containing the dominant signals) for superharmonic imaging.

In previous work, ${ }^{14}$ superharmonic imaging has been compared to second harmonic imaging, both using simulations and experiments. However, in this comparison the frequency ranges of the second harmonic and the dominant superharmonic components differ, so it is assumed that the modalities are not compared under their optimal conditions for the same application. Also, the simulations are performed for a less realistic situation involving water and an axially symmetric transducer and corresponding ultrasound field. The axial symmetry also appears in other work. ${ }^{8}$ Moreover, the latter paper mainly compares the superharmonic and second harmonic components around the focus. Zemp et al. ${ }^{16}$ compared fundamental and second harmonic imaging at their optimal frequencies, but only investigated the field around the focus and only showed normalized results. To the authors' knowledge, no research has been published that compares all of the relevant features of fundamental, second harmonic, and superharmonic imaging at their optimal frequencies for a realistic application, i.e., involving phased array transducers, a medium with tissue-like attenuation, and a maximum allowable mechanical index (MI). In view of the nonlinear character of the physics that underlies harmonic imaging, comparison of the modalities under the desired realistic circumstances cannot be deduced from the results obtained under different circumstances. The aim of this paper is to make a fair comparison of fundamental, second harmonic, and superharmonic imaging under conditions that resemble a realistic medical diagnostic situation. The study is performed using numerical simulations, and the chosen situation applies to echocardiography. For each modality, the realistic situation is mirrored in all aspects, e.g., by using the optimal transmission frequencies, different phased array topologies, equal focal distances, the highest allowable pressures in transmission, and tissue-like attenuation. The three imaging modalities will be evaluated with regard to the lateral beam shape over the entire imaging depth, axial pulse shape, and pulse-echo axial pressure.

A truly fair comparison between different harmonic imaging modalities can only be performed by a numerical study, such as presented by Zemp et al. ${ }^{16}$ The experimental comparison of these modalities would be very difficult, because it is impossible to obtain suitable phased array transducers with the same aperture size, the correct center frequency, equal relative bandwidths, and equal transmit efficiencies. Deviations form the carefully selected transducer parameters would result in an unfair comparison. Both the INCS method ${ }^{17-19}$ and a KZK implementation ${ }^{20-22}$ are used for the numerical simulations in this study. The KZK implementation was adapted to handle frequency power-law losses, since the accurate modeling of tissue-like attenuation, next to nonlinearity, is important in the simulations. The INCS method is also able to deal with these kinds of losses. 
A description of both nonstandard simulation tools will be given to show their interrelation and to enable reproduction of the results.

\section{METHOD}

\section{A. Westervelt equation}

The nonlinear propagation of the ultrasound field in a source-free region of a thermoviscous fluid may be described by the source-free Westervelt equation ${ }^{22,23}$

$$
\nabla^{2} p-\frac{1}{c_{0}^{2}} \frac{\partial^{2} p}{\partial t^{2}}+\frac{\delta}{c_{0}^{4}} \frac{\partial^{3} p}{\partial t^{3}}=-\frac{\beta}{\rho_{0} c_{0}^{4}} \frac{\partial^{2} p^{2}}{\partial t^{2}} .
$$

Here, the ultrasound field is represented by the acoustic pressure $p$, and the medium is represented by the ambient density of mass $\rho_{0}$, the ambient speed of sound $c_{0}$, the diffusivity of sound $\delta$, and the coefficient of nonlinearity $\beta$. The latter quantity is often written as

$$
\beta=1+\frac{B}{2 A},
$$

where $B / A$ is the parameter of nonlinearity, which follows from the second order Taylor expansion of the state equation of the medium around equilibrium. In all media that show attenuation, a plane propagating ultrasound wave decays exponentially with distance. For a thermoviscous medium, the corresponding attenuation coefficient satisfies the quadratic frequency $\operatorname{law}^{22,24} \alpha=\alpha_{0}|\omega|^{2}$. Experiments show that most biomedical tissues exhibit a more general frequency power law $^{25,26} \alpha=\alpha_{0}|\omega|^{\alpha_{i}}$, with $1 \leq \alpha_{1} \leq 2$. To accommodate this kind of attenuation as well, Eq. (1) is generalized to ${ }^{17,18,27}$

$$
\nabla^{2} p-\frac{1}{c_{0}^{2}} \frac{\partial^{2}\left(\chi *_{t} p\right)}{\partial t^{2}}=-\frac{\beta}{\rho_{0} c_{0}^{4}} \frac{\partial^{2} p^{2}}{\partial t^{2}} .
$$

Here, $\chi=\chi(\mathrm{t})$ is a causal relaxation function, and ${ }_{t}$ denotes a temporal convolution. The relaxation function may be split according to

$$
\chi(t)=\delta(t)+a(t)
$$

where $\delta(t)$ is the Dirac delta function, which represents the instantaneous behavior of the medium, and $a(t)$ is a causal memory function, which is associated with the attenuation and dispersion of the medium.

\section{B. KZK simulations}

The far field behavior of the considered unsteered beams will be simulated using an approximate method. For these beams most of the ultrasound energy propagates in the positive $z$-direction, i.e. in the direction that is normal to and away from the transducer. By introducing the retarded time $T=t-z / c_{0}$ and neglecting the resulting derivatives with respect to $z$ (parabolic approximation), Eq. (3) may be turned into a beam equation or evolution equation that favors the positive $z$-direction. The resulting equation is

$$
\frac{\partial^{2} \bar{p}}{\partial \tau \partial z}-\frac{c_{0}}{2} \nabla_{\perp}^{2} \bar{p}+\frac{1}{2 c_{0}} a(\tau) *_{\tau} \frac{\partial^{2} \bar{p}}{\partial \tau^{2}}=\frac{\beta}{2 \rho_{0} c_{0}^{3}} \frac{\partial^{2} \bar{p}^{2}}{\partial \tau^{2}}
$$

In the retarded time frame, the acoustic pressure is represented by $\bar{p}(x, y, z, \tau)=p(x, y, z, t)$, while the memory function remains unchanged. Apart from the more general loss term, Eq. (5) is identical to the Khokhlov-Zabolotskaya-Kuznetsov (KZK) equation ${ }^{20-22}$ for a nonlinear medium with thermoviscous behavior. The numerical solution of this equation will be based on the method proposed by Lee and Hamilton $^{28}$ for circular transducers, and in particular on the extension by Yang and Cleveland ${ }^{29}$ for rectangular transducers. This involves the introduction of the dimensionless quantities

$$
\begin{aligned}
& X=\frac{x}{a}, \quad Y=\frac{y}{b}, \quad Z=\frac{z}{d}, \\
& T=\omega_{0} \tau, \\
& P(X, Y, Z, T)=\frac{\bar{p}(x, y, z, \tau)}{p_{0}},
\end{aligned}
$$

where $d$ is a characteristic length in the direction of propagation (e.g., the focal distance), $a$ and $b$ are the lateral dimensions of the sound source, $\omega_{0}$ is a characteristic angular frequency (e.g., the center frequency of the transmitted pulse) and $p_{0}$ is the peak pressure of the sound wave at the source. Subsequent integration with respect to $\tau$ gives

$$
\begin{aligned}
\frac{\partial P}{\partial Z}= & \frac{1}{4} \int_{-\infty}^{T}\left(\frac{1}{G_{x}} \frac{\partial^{2} P}{\partial X^{2}}+\frac{1}{G_{y}} \frac{\partial^{2} P}{\partial Y^{2}}\right) d T^{\prime} \\
& +N P \frac{\partial P}{\partial T}+A *_{T} \frac{\partial P}{\partial T},
\end{aligned}
$$

in which the following quantities appear

$$
\begin{aligned}
& G_{x}=\frac{\omega_{0} a^{2}}{2 c_{0} d}, \quad G_{y}=\frac{\omega_{0} b^{2}}{2 c_{0} d}, \\
& N=\frac{\beta \omega_{0} p_{0} d}{\rho_{0} c_{0}^{3}}, \\
& A(T)=-\frac{d}{2 c_{0}} a\left(\omega_{0}^{-1} T\right) .
\end{aligned}
$$

The numerical solution of Eq. (9) is performed by the algorithms described by Voormolen. ${ }^{31}$ This involves the traditional operator splitting scheme, ${ }^{28,32}$ which separately accounts for the effects of diffraction (the integral term), nonlinearity (the term with the factor $N$ ) and attenuation (the term with the factor $A$ ). The diffraction substep is performed in the time domain and uses the implicit backward finite difference (IBFD) method $^{33}$ in the near-field and the alternating direction implicit (ADI) finite difference scheme at larger axial distances. The nonlinearity substep is also performed in the time domain and employs a time base transformation. The attenuation substep is performed in the frequency domain to avoid the explicit evaluation of the convolution in the time domain. 
In case of general frequency power law losses of the form $\alpha=\alpha_{0}|\omega|^{\alpha_{1}}$, one can combine Eqs. (3) and (4) of this paper, and compare the result with Eqs. (B6) and (B7) of Szabo. ${ }^{30}$ Using generalized calculus in the same sense as in that paper, it may be deduced that

$$
a(t)=\frac{4 c_{0} h H(t)}{\alpha_{1}\left(\alpha_{1}+1\right) t^{\alpha_{1}}},
$$

in which $h=-\alpha_{0} \Gamma\left(\alpha_{1}+2\right) \cos \left[\left(\alpha_{1}+1\right) \pi / 2\right] / \pi$ with $\Gamma$ being the gamma function, and $H(t)$ is the Heaviside step function. Application of Eqs. (12) and (13) gives for the attenuation term in Eq. (9)

$$
A_{*_{T} T} \frac{\partial P}{\partial T}=\frac{2 d \omega_{0}^{\alpha_{1}} h}{\alpha_{1}+1} \int_{0}^{T} \frac{P\left(T^{\prime}\right)}{\left(T-T^{\prime}\right)^{\alpha_{1}+1}} d T^{\prime},
$$

where it has been used that $P(T)=0$ for $T<0$. For the evaluation of this term, it is employed that in the generalized sense a convolution in the time domain still corresponds to a multiplication in the frequency domain, and hence

$$
A_{{ }^{*} T} \frac{\partial P}{\partial T}=\mathbf{F}^{-1}\{\mathbf{F}[P(T)] \mathbf{F}[K(T)]\} .
$$

Here, $\mathbf{F}$ and $\mathbf{F}^{-1}$ denote the forward and inverse Fourier transformation, respectively, and the kernel function $K(T)$ is given by

$$
K(T)=\frac{2 d \omega_{0}^{\alpha_{1}} h H(T)}{\left(\alpha_{1}+1\right) T^{\alpha_{1}}+1} .
$$

The Fourier transformation of this function is

$$
\mathbf{F}[K(T)]=d \omega_{0}^{\alpha_{1}} \alpha_{0}(j \Omega)^{\alpha_{1}},
$$

where $\Omega$ is the angular frequency associated with the time $T$. This expression is directly applied in Eq. (15), which is implemented using fast Fourier transforms.

\section{INCS simulations}

Close to the source it cannot be assumed a priori that most of the ultrasound energy propagates in a preferred direction, and the KZK equation may become inaccurate. Therefore, the directionally independent Iterative nonlinear contrast source (INCS) method ${ }^{17-19}$ will be used for the simulation of the near field of the beams. This method is based on the generalized Westervelt equation

$$
\nabla^{2} p-\frac{1}{c_{0}^{2}} \frac{\partial^{2}\left(\chi *_{t} p\right)}{\partial t^{2}}=-S^{N L}(p)-S,
$$

where, in accordance with Eq. (3), the nonlinear term equals

$$
S^{N L}(p)=\frac{\beta}{\rho_{0} c_{0}^{4}} \frac{\partial^{2} p^{2}}{\partial t^{2}} .
$$

The additional term $S$ is the primary source term that represents the transducer. In the absence of $S^{\mathrm{NL}}(p)$, the primary source generates the linear contribution to the wave field. With the INCS method, $S^{\mathrm{NL}}(p)$ gets the role of a separate source. This source accounts for the difference between the linear and the nonlinear case, i.e., it generates the nonlinear contribution to the wave field, and is called a contrast source. The contrast source depends on the field itself, and is distributed over the entire space. Since the wave operator at the left hand side of Eq. (18) applies to a linear and attenuative medium, both the primary source and the nonlinear contrast source may be thought to operate in a linear and attenuative background medium. This implies that the fields of the primary source and the contrast source may be computed using the same techniques that apply to a linear medium. Now suppose that the Green's function of the background medium, i.e., the function $G$ that satisfies

$$
\nabla^{2} G-\frac{1}{c_{0}^{2}} \frac{\partial^{2}\left(\chi *_{t} G\right)}{\partial t^{2}}=-\delta(x) \delta(y) \delta(z) \delta(t),
$$

is known. This function may most conveniently be derived in the space-frequency domain. ${ }^{17,18}$ Because the Green's function describes the spatiotemporal impulse response of the relevant medium, the solution of Eq. (18) may formally be written as

$$
p=G *_{x, y, z, t}\left[S+S^{N L}(p)\right]
$$

Here, $*_{x, y, z, t}$ denotes a convolution over the entire spatial and temporal domain where either source term has a significant value. Since the nonlinear contrast source depends on the total pressure wave field $p$, the equation yields an implicit solution. The main assumption of the INCS method is that for weakly to moderately nonlinear behavior, as encountered with diagnostic ultrasound, the contribution of the contrast source will be relatively small. In that case, increasingly accurate approximations of the total pressure wave field may be obtained by first computing the linear field contribution $p^{(0)}$, using this to compute an approximation of the contrast source, then computing an approximation of the nonlinear field, and successively repeating of the last two steps. This results in the iterative Neumann scheme

$$
\begin{aligned}
p^{(0)} & =G *_{x, y, z, t} S, \\
p^{(j)} & =G *_{x, y, z, t}\left[S+S^{N L}\left(p^{(j-1)}\right)\right] \\
& =p^{(0)}+G *_{x, y, z, t} S^{N L}\left(p^{(j-1)}\right)(j \geq 1) .
\end{aligned}
$$

As a rule of thumb, in the $j$ th approximation the fundamental up to the $(j-1)$ th harmonic will be represented accurately. The most involving task in the numerical evaluation of the scheme is the spatiotemporal convolution, which is efficiently performed with the filtered convolution method. ${ }^{34}$ The method first filters out all spatial and temporal frequencies above the highest frequencies of interest. This enables sampling at only two points per shortest period or shortest wavelength, without introducing aliasing. Then the resulting four-dimensional discrete convolution is performed using Fast Fourier Transforms. To model frequency power law losses of 
the form $\alpha=\alpha_{0}|\omega|^{\alpha_{1}}$, the INCS method employs a relaxation function $x$ that is defined in the Laplace domain as ${ }^{17,18,27}$

$$
\hat{\chi}(s)=\left(1+\frac{c_{0} \alpha_{0} s^{\alpha_{1}-1}}{\cos \left(\pi \alpha_{1} / 2\right)\left[1+\left(s / s_{1}\right)^{a}\right]}\right)^{2},
$$

where $a$ is an integer with value $a>\alpha_{1}-1$ and $s_{1}$ is taken greater than the highest angular frequency of interest. The term $\left(s / s_{1}\right)^{a}$ is necessary for causality purposes, but has no influence for the angular frequencies of interest. For low losses, the quadratic term in the expansion of Eq. (24) may be omitted. Under these circumstances, comparison of Eq. (24) with the transformed version of Eq. (4) shows that

$$
\hat{a}(s)=\frac{2 c_{0} \alpha_{0} s^{\alpha_{1}-1}}{\cos \left(\pi \alpha_{1} / 2\right)} .
$$

The inverse Laplace transformation of this function is identical to $a(t)$ in Eq. (13). From this it may be concluded that for low losses and angular frequencies well below $s_{1}$, the attenuation in the KZK and INCS simulations is the same.

\section{SIMULATION SETTINGS}

In this paper three imaging modalities applied to echocardiography are compared: fundamental, second, and superharmonic imaging. The acoustic beams produced in each modality are calculated from the source plane up to an axial depth of $15 \mathrm{~cm}$ using a full 3D implementation of the KZK equation. However, only the results from $6-15 \mathrm{~cm}$ are analyzed. The near-fields of these beams are calculated and analyzed from the source plane up to an axial depth of $\sim 7 \mathrm{~cm}$ using the INCS method. To estimate the pressure received by the transducer for each of the imaging modalities in pulse-echo mode, linear back propagation is assumed. The scatterer is assumed to be small, and situated on the propagation axis. It is further assumed that the field at the scatterer is totally reflected in the direction of the transducer, where it is registered. No receive focusing is applied.

The transmission pressure amplitude $\left(p_{0}\right)$ at the transducer element surface is taken such that a Mechanical Index (MI) of 1.9 is produced in water ${ }^{25}$ (the MI is defined as the peak negative pressure divided by the square root of the frequency in $\mathrm{MHz}$ ). The lateral and elevation focus is set to 9 $\mathrm{cm}$ and no beam steering is applied. Three cycle Gaussian apodized sine bursts, with a center frequency equal to the respective optimal transmission frequency (see section I), are used as transmission signals.

The transducer configurations used to simulate each imaging modality are summarized in Table I. Notice that the footprint for each array is equal, i.e., $16 \mathrm{~mm} \times 22 \mathrm{~mm}$. The transducer geometry used for superharmonic imaging is based on a previously reported interleaved array, ${ }^{15}$ which consists of a low frequency subarray interleaved with a high frequency subarray. The low frequency subarray is used in transmission, and the high frequency subarray is used in reception. Both subarrays consist of 44 elements each, and the subarray pitch is $0.5 \mathrm{~mm}$.
TABLE I. The array geometries used for the simulations.

\begin{tabular}{lcccccc}
\hline \hline & $\begin{array}{c}\text { Transmit } \\
\text { freq. } \\
(\mathrm{MHz})\end{array}$ & $\begin{array}{c}\text { po } \\
(\mathrm{kPa})\end{array}$ & $\begin{array}{c}\text { Footprint } \\
\left(\mathrm{mm}^{2}\right)\end{array}$ & $\begin{array}{c}\text { No. } \\
\text { elements } \\
-\end{array}$ & $\begin{array}{c}\text { of Element size } \\
\left(\mathrm{mm}^{2}\right)\end{array}$ & $\begin{array}{c}\text { Pitch } \\
(\mathrm{mm})\end{array}$ \\
\hline FI & 3.5 & 774 & $16 \times 22$ & 128 & $16 \times 0.12$ & 0.17 \\
THI & 1.8 & 910 & $16 \times 22$ & 88 & $16 \times 0.20$ & 0.25 \\
SHI & 1.2 & 1730 & $16 \times 22$ & 44 & $16 \times 0.20$ & 0.50 \\
\hline \hline
\end{tabular}

To evaluate the axial resolution in the case of superharmonic imaging the dual-pulse method ${ }^{35,36}$ is applied. This technique is required to prevent ghosting artifacts associated with imaging based on multiple harmonics and using temporally wide (i.e., spectrally narrow) transmission pulses. This imaging protocol is based on the transmission of two pulses with a slightly different center frequency. Subsequently, both time traces are summed to create an image. The frequencies of the first and second pulses are 1.1 and $1.3 \mathrm{MHz}$, and were determined using the methodology described by van Neer et $a l .{ }^{36}$ The tissue parameters are based on those reported for cardiac tissue: ${ }^{25} B / A=5.8, \alpha_{0}=0.52 \mathrm{~dB} \cdot \mathrm{cm}^{-1} \cdot \mathrm{MHz}^{-1}$, $p_{0}=1060 \mathrm{~kg} / \mathrm{m}^{3}$ and $c_{0}=1529 \mathrm{~m} / \mathrm{s}$. In this case $\alpha_{1}=1$, but for the INCS simulations, a slightly larger value is used to avoid a zero denominator in Eq. (4). To determine $p_{0}$, simulations are performed using the properties of water: ${ }^{25}$ $B / A=4.96, \quad \alpha_{0}=2.17 \times 10^{-3} \mathrm{~dB} \cdot \mathrm{cm}^{-1} \cdot \mathrm{MHz}^{-2}, \quad \rho_{0}=998.2$ $\mathrm{kg} / \mathrm{m}^{3}$ and $c_{0}=1482.3 \mathrm{~m} / \mathrm{s}$.

\section{RESULTS}

\section{A. Lateral beam profiles}

Figures 1-5 display the normalized lateral pressure profiles of the fundamental at $3.5 \mathrm{MHz}$, the second harmonic at 3.6 MHz, and the superharmonic at 3.6-6.0 MHz at axial depths of $3,6,9,12$, and $15 \mathrm{~cm}$. The profiles have been calculated using the INCS method (gray lines in Figs. 1 and 2) and KZK simulations (black lines in Figs. 2-5). The INCS and $\mathrm{KZK}$ results are in good agreement. The differences between the results of both methods are between $0-1 \mathrm{~dB}$,

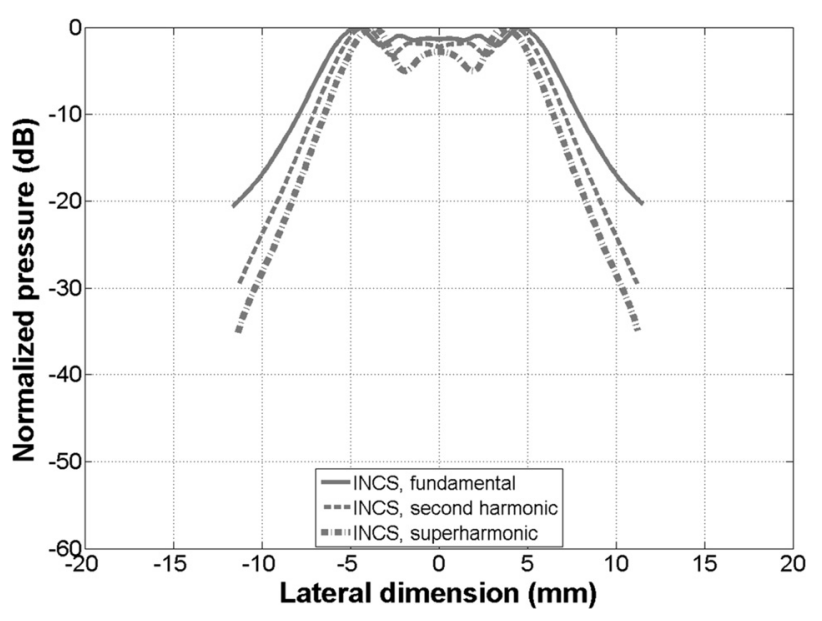

FIG. 1. The normalized lateral pressure profiles of the fundamental at 3.5 MHz (solid line), the second harmonic at $3.6 \mathrm{MHz}$ (dashed line) and the superharmonic at 3.6-6.0 MHz (dash-dotted line) at an axial depth of $3 \mathrm{~cm}$. 


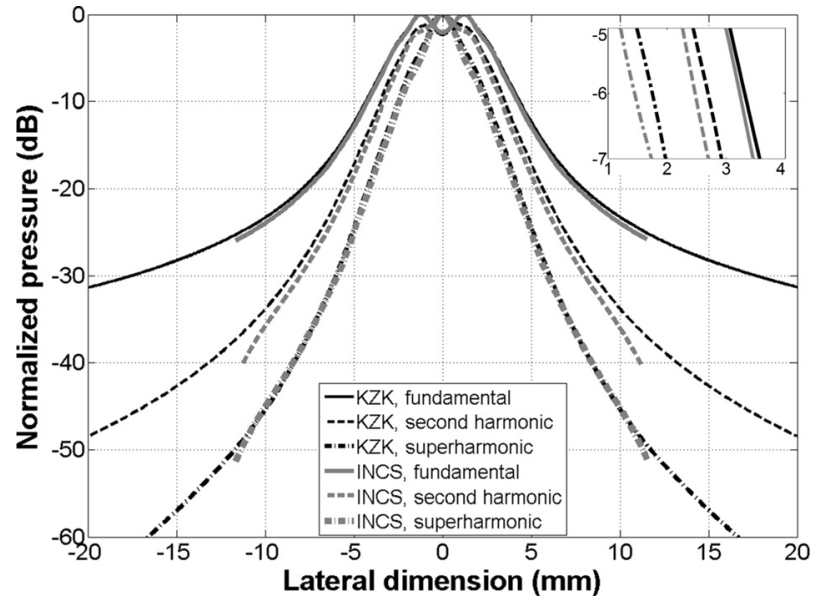

FIG. 2. The normalized lateral pressure profiles of the fundamental at $3.5 \mathrm{MHz}$ (solid line), the second harmonic at $3.6 \mathrm{MHz}$ (dashed line) and the superharmonic at 3.6-6.0 MHz (dash-dotted line) at an axial depth of $6 \mathrm{~cm}$.

$0-2 \mathrm{~dB}$, and $0-1 \mathrm{~dB}$ for the fundamental, second harmonic and superharmonic, respectively. The lateral $-6 \mathrm{~dB}$ beam widths and beam roll-off at particular off-axis distances are given in Table II. Except for an axial depth of $3 \mathrm{~cm}$, the values in this table are taken from the KZK simulations.

\section{B. Axial pulse shape}

The normalized envelopes of the pressure pulses produced at focus by the fundamental at $3.5 \mathrm{MHz}$ (solid line), the second harmonic at $3.6 \mathrm{MHz}$ (dashed line), and the superharmonic at 3.6-6.0 MHz (dash-dotted line) are displayed in Fig. 6(a) on a linear scale. Figure 6(b) shows these envelopes in decibel. The envelopes have been calculated using KZK simulations. The axial lengths produced by each modality at the $-6,-10$, and $-20 \mathrm{~dB}$ levels are given in Table III.

\section{Pulse-echo axial pressure}

Figure 7 displays the pulse-echo axial pressure profiles of the fundamental at $3.5 \mathrm{MHz}$ (solid line), the second

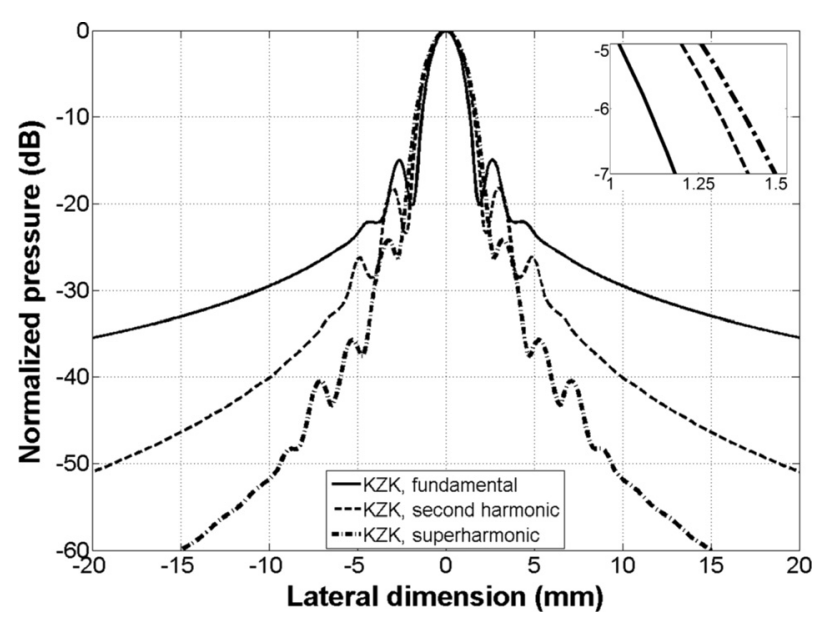

FIG. 3. The normalized lateral pressure profiles of the fundamental at $3.5 \mathrm{MHz}$ (solid line), the second harmonic at $3.6 \mathrm{MHz}$ (dashed line) and the superharmonic at 3.6-6.0 MHz (dash-dotted line) at an axial depth of $9 \mathrm{~cm}$.

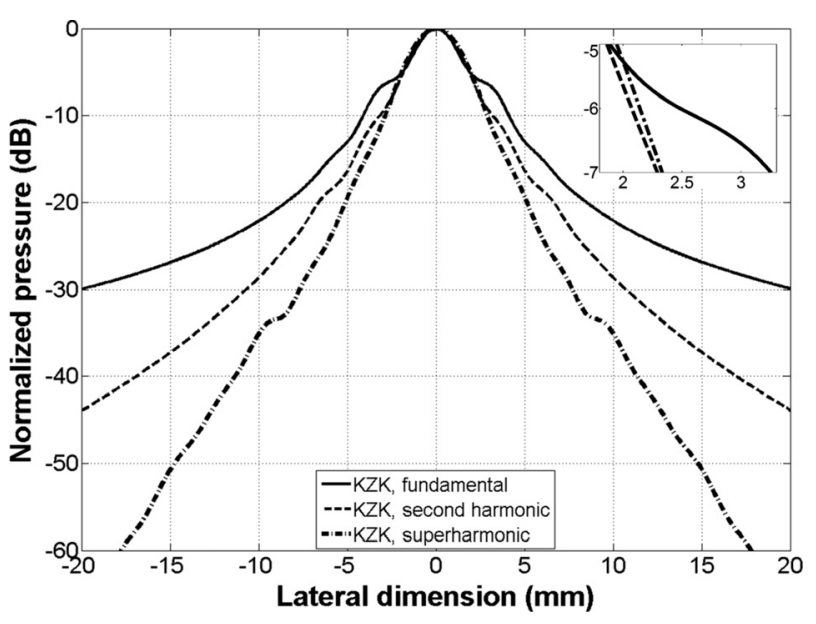

FIG. 4. The normalized lateral pressure profiles of the fundamental at 3.5 MHz (solid line), the second harmonic at $3.6 \mathrm{MHz}$ (dashed line) and the superharmonic at 3.6-6.0 MHz (dash-dotted line) at an axial depth of $12 \mathrm{~cm}$.

harmonic at 3.6 MHz (dashed line), and the superharmonic at 3.6-6.0 MHz (dash-dotted line). The profiles have been calculated using the INCS method (gray color) and using KZK simulations (black color). The INCS and KZK results are in good agreement. The differences between the results of both methods are between $0-0.5 \mathrm{~dB}, 0-2 \mathrm{~dB}$, and $0-1$ $\mathrm{dB}$ for the fundamental, second harmonic, and superharmonic, respectively. The focal spot is at $70 \mathrm{~mm}$ for the fundamental beam, at $66 \mathrm{~mm}$ for the second harmonic, and at $65 \mathrm{~mm}$ for the superharmonic (values taken from KZK simulations). The pulse-echo axial intensities produced by each modality in the near-field at $1 \mathrm{~cm}$ (from INCS simulations), the focal point (from KZK simulations) and the far-field at $15 \mathrm{~cm}$ (from KZK simulations) are shown in Table III.

\section{DISCUSSION}

\section{A. Lateral resolution and roll-off}

To the authors' knowledge the comparison between the fundamental, second harmonic, and superharmonic imaging

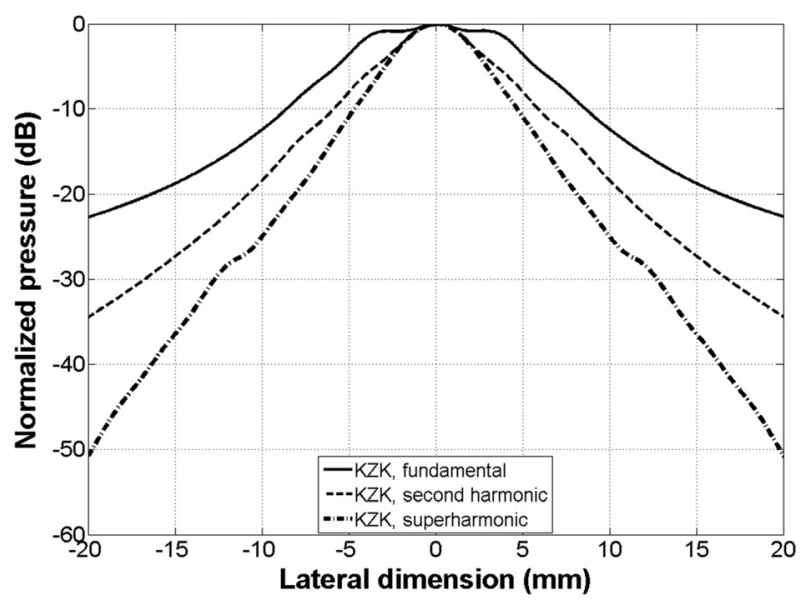

FIG. 5. The normalized lateral pressure profiles of the fundamental at $3.5 \mathrm{MHz}$ (solid line), the second harmonic at $3.6 \mathrm{MHz}$ (dashed line) and the superharmonic at $3.6-6.0 \mathrm{MHz}$ (dash-dotted line) at an axial depth of $15 \mathrm{~cm}$. 
TABLE II. Lateral beam characteristics.

\begin{tabular}{|c|c|c|c|}
\hline & Fundamental & 2nd harmonic & Superharmonic \\
\hline Transmit frequency & $3.5 \mathrm{MHz}$ & $1.8 \mathrm{MHz}$ & $1.2 \mathrm{MHz}$ \\
\hline Receive frequency & $3.5 \mathrm{MHz}$ & $3.6 \mathrm{MHz}$ & $3.6-6.0 \mathrm{MHz}$ \\
\hline \multicolumn{4}{|l|}{ Axial depth $3 \mathrm{~cm}$} \\
\hline$-6 \mathrm{~dB}$ width $(\mathrm{mm})$ & 13.8 & 12.5 & 11.4 \\
\hline Roll-off at $10 \mathrm{~mm}(\mathrm{~dB})$ & -17 & -24 & -28 \\
\hline \multicolumn{4}{|l|}{ Axial depth $6 \mathrm{~cm}$} \\
\hline$-6 \mathrm{~dB}$ width (mm) & 6.6 & 5.4 & 3.5 \\
\hline Roll-off at $15 \mathrm{~mm}(\mathrm{~dB})$ & -28 & -43 & -57 \\
\hline \multicolumn{4}{|l|}{ Axial depth $9 \mathrm{~cm}$} \\
\hline$-6 \mathrm{~dB}$ width $(\mathrm{mm})$ & 2.2 & 2.6 & 2.8 \\
\hline Roll-off at $10 \mathrm{~mm}(\mathrm{~dB})$ & -30 & -40 & -52 \\
\hline Roll-off at $15 \mathrm{~mm}(\mathrm{~dB})$ & -33 & -46 & -60 \\
\hline \multicolumn{4}{|l|}{ Axial depth $12 \mathrm{~cm}$} \\
\hline$-6 \mathrm{~dB}$ width $(\mathrm{mm})$ & 5.0 & 4.2 & 4.3 \\
\hline Roll-off at $15 \mathrm{~mm}(\mathrm{~dB})$ & -27 & -37 & -51 \\
\hline \multicolumn{4}{|l|}{ Axial depth $15 \mathrm{~cm}$} \\
\hline-6 dB width $(\mathrm{mm})$ & 12.6 & 8.1 & 6.5 \\
\hline Roll-off at $15 \mathrm{~mm}(\mathrm{~dB})$ & -19 & -27 & -36 \\
\hline
\end{tabular}

modalities at their respective optimal frequencies is the first of its kind. The $-6 \mathrm{~dB}$ lateral beam widths of the second and superharmonic are $18 \%$ and $27 \%$ wider than the fundamental
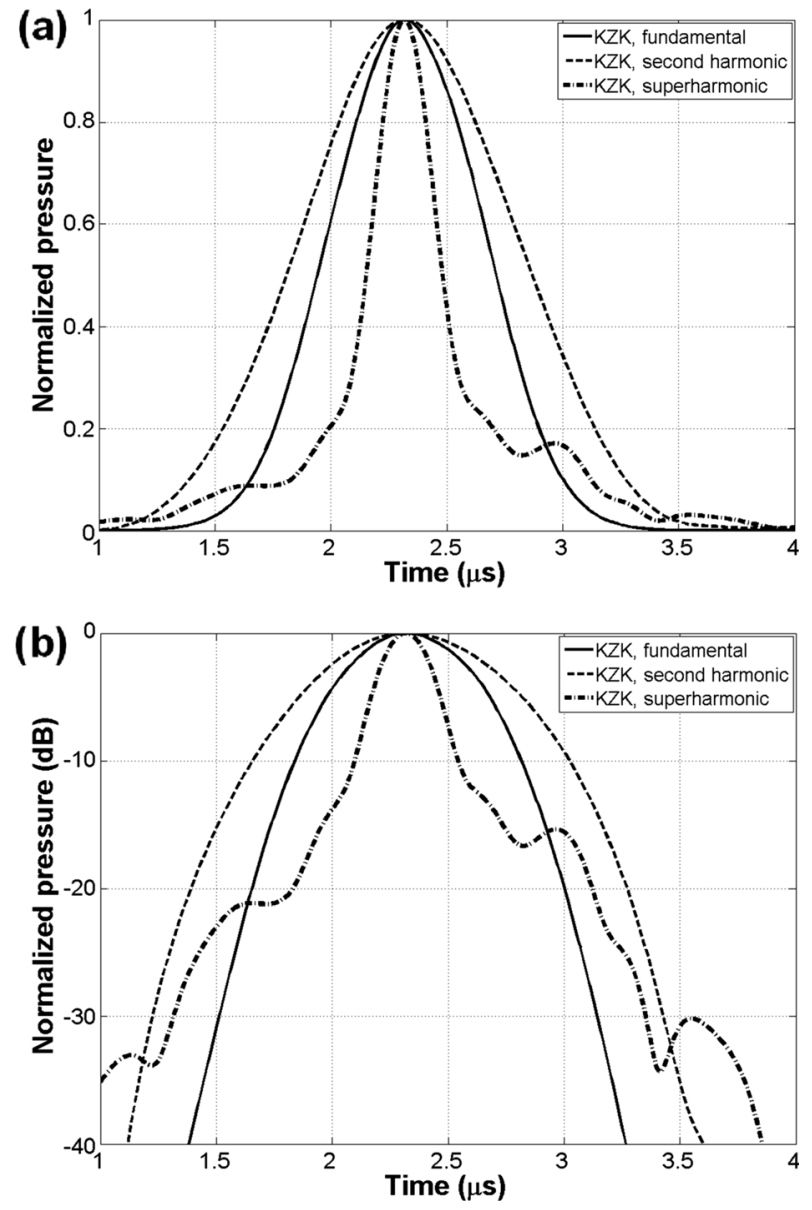

FIG. 6. (a) The normalized envelopes of the pressure pulses produced at focus by the fundamental at $3.5 \mathrm{MHz}$ (solid line), the second harmonic at 3.6 MHz (dashed line) and the superharmonic at 3.6-6.0 $\mathrm{MHz}$ (dash-dotted line) displayed on a linear scale. (b) The normalized envelopes displayed in decibel.
TABLE III. Axial lengths and pulse-echo intensities.

\begin{tabular}{|c|c|c|c|}
\hline $\begin{array}{l}\text { Transmit frequency } \\
\text { Receive frequency }\end{array}$ & $\begin{array}{c}\text { Fundamental } \\
\begin{array}{c}3.5 \mathrm{MHz} \\
3.5 \mathrm{MHz}\end{array}\end{array}$ & $\begin{array}{c}\text { 2nd harmonic } \\
\begin{array}{c}1.8 \mathrm{MHz} \\
3.6 \mathrm{MHz}\end{array}\end{array}$ & $\begin{array}{c}\text { Superharmonic } \\
1.2 \mathrm{MHz} \\
3.6-6.0 \mathrm{MHz}\end{array}$ \\
\hline \multicolumn{4}{|l|}{ Axial length } \\
\hline$-6 \mathrm{~dB}(\mu \mathrm{s})$ & 0.77 & 1.1 & 0.33 \\
\hline$-10 \mathrm{~dB}(\mu \mathrm{s})$ & 0.98 & 1.37 & 0.45 \\
\hline$-20 \mathrm{~dB}(\mu \mathrm{s})$ & 1.3 & 1.9 & 1.3 \\
\hline \multicolumn{4}{|l|}{ Axial Pressure } \\
\hline Near-field at $1 \mathrm{~cm}(\mathrm{~dB})$ & 112 & 87 & 59 \\
\hline Focus $(\mathrm{dB})$ & 106 & 101 & 93 \\
\hline Far-field at $15 \mathrm{~cm}(\mathrm{~dB})$ & 68 & 67 & 61 \\
\hline
\end{tabular}

at the geometric focus (an axial distance of $9 \mathrm{~cm}$ ). Similar results have been reported for the second harmonic by Zemp et al. ${ }^{16}$ They compared simulations of $4 \mathrm{MHz}$ fundamental beams and 2-4 MHz second harmonic beams produced by phased array transducers.

At short axial distance $(6 \mathrm{~cm})$ the superharmonic beam has a $-6 \mathrm{~dB}$ lateral beamwidth, which is $47 \%$ and $35 \%$ narrower than the fundamental and second harmonic beams, respectively. At a large axial distance $(15 \mathrm{~cm})$ the superharmonic beam has a $48 \%$ and $36 \%$ narrower beamwidth than the fundamental and second harmonic beams, respectively. This is caused by the fact that for the fundamental of the superharmonic beam the natural focus of the aperture is close to the lateral and elevation foci $(9 \mathrm{~cm})$. Therefore, the focal zone is elongated and the fundamental ultrasound beam spreads slowly. The resulting superharmonic beam shape is even narrower due to the autofocusing effect of nonlinear propagation. The fundamental of the second harmonic beam is more focused than the fundamental of the superharmonic, but less so compared to the fundamental beam. In case of the second harmonic beam, the autofocusing effect is also weaker than for the superharmonic beam. The autofocusing of second harmonic beams in the far-field was also reported by Tranquart et al. ${ }^{1}$

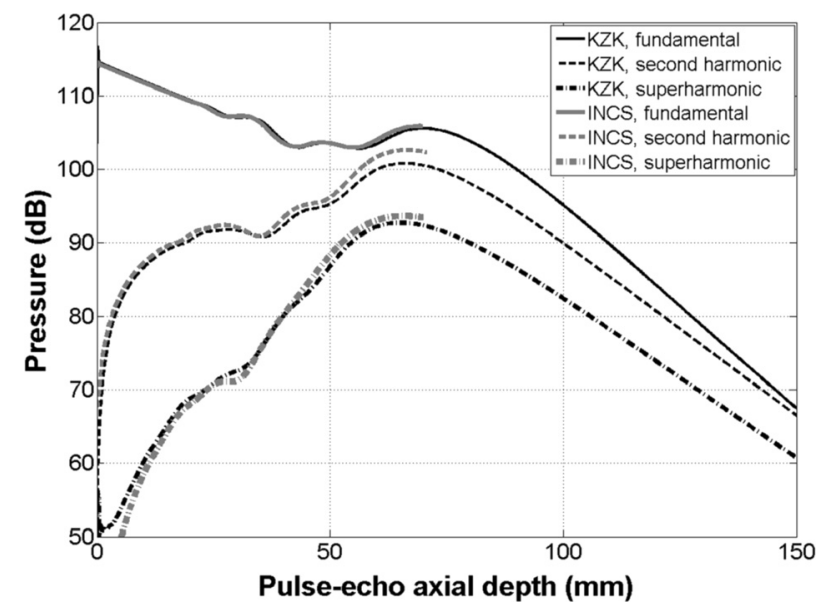

FIG. 7. The pulse-echo axial pressure profiles of the fundamental at $3.5 \mathrm{MHz}$ (solid line), the second harmonic at $3.6 \mathrm{MHz}$ (dashed line) and the superharmonic at 3.6-6.0 MHz (dash-dotted line). The pressure values are relative to $1 \mathrm{~Pa}$. 
Usually, in the literature the lateral profile around focus is discussed, where sidelobes are clearly discernable (as can be seen from Fig. 3). If lateral profiles away from focus are discussed (such as Figs. 1 and 5, but to a lesser extent also Figs. 2 and 4), the sidelobes are not or not clearly visible. Therefore, the roll-off is chosen to describe and compare the lateral beam behavior. The roll-off of the second and superharmonic beams is considerably higher than that of the fundamental beam. For example, around the geometric focus ( 9 $\mathrm{cm}$ ) the second harmonic pressure at $10 \mathrm{~mm}$ off-axis was 10 $\mathrm{dB}$ lower than that of the fundamental, which was similar to the $12 \mathrm{~dB}$ difference reported in the work of Zemp et al. ${ }^{16}$ Over the axial interval $6-15 \mathrm{~cm}$, the roll-off of the second harmonic beam as characterized by the pressure at $15 \mathrm{~mm}$ off-axis was 8-15 dB higher than that of the fundamental. Over the same axial interval, the improvement in roll-off of the superharmonic beam relative to that of the second harmonic beam was 9-14 $\mathrm{dB}$, which is approximately equal to the improvement in roll-off of the second harmonic beam compared to the fundamental beam, and amounts to an improvement of 17-29 dB in the superharmonic beam relative to the fundamental beam. The improved roll-off of the second harmonic and superharmonic beams is also caused by the autofocusing effect of nonlinear propagation.

\section{B. Axial resolution}

The axial length of the fundamental pulse is $28 \%$ shorter at the $-10 \mathrm{~dB}$ level and $28 \%$ shorter at the $-20 \mathrm{~dB}$ level than the length of the second harmonic signal. The superharmonic axial length (based on the dual-pulse method) is in turn $67 \%$ shorter at the $-10 \mathrm{~dB}$ level and $31 \%$ shorter at the $-20 \mathrm{~dB}$ level than that of the second harmonic. These last results are similar to the $62 \%$ decrease in axial pulse length for the superharmonic relative to the second harmonic reported by Bouakaz and de Jong. ${ }^{8}$ The difference is explained by the fact that Bouakaz and de Jong simulated a circular symmetric transducer in stead of a rectangular transducer, and used a 1.7 $\mathrm{MHz}$ transmission frequency for the second harmonic compared to the $1.8 \mathrm{MHz}$ transmission frequency used here.

The axial length of the simulated superharmonic component obtained using the dual-pulse method is about $35 \%$ shorter compared to previously reported experimental results. ${ }^{36}$ Part of this discrepancy is caused by the difference in transmission frequencies, which were 0.9 and $1.15 \mathrm{MHz}$ in the experiments and which are 1.1 and $1.3 \mathrm{MHz}$ here. Also, the previously reported results were obtained using a phased array transducer combined with an ultrasound system. The pulse created by the arbitrary waveform generators was affected (in practice lengthened) by the transfer function of both the transducer and the electrical circuitry, effects which were not taken into account in the simulations reported here.

\section{Pulse-echo axial pressure: Depth-of-field}

The pulse-echo near-field pressure of the superharmonic beam at an axial distance of $1 \mathrm{~cm}$ is $34 \mathrm{~dB}$ below its pressure at focus. For the fundamental and second harmonic beams the near-field intensities are $6 \mathrm{~dB}$ above and $14 \mathrm{~dB}$ below their respective intensities at focus. The pressure at focus of the superharmonic beam is 13 and $8 \mathrm{~dB}$ lower than those of the fundamental and second harmonic beams, respectively. However, the pressure loss associated with the imaging at larger depths is lower for superharmonic imaging compared to fundamental and second harmonic imaging. At $15 \mathrm{~cm}$ the superharmonic pulse-echo pressure is 7 and $6 \mathrm{~dB}$ lower than that of the fundamental and second harmonic, respectively. These results mean that the depth-of-field of superharmonic imaging is lower than those of fundamental and second harmonic imaging. If the second harmonic pressure at $15 \mathrm{~cm}$ is taken as a minimum threshold pressure, the second harmonic depth-offield stretches from 0.1 to $15 \mathrm{~cm}$, while the superharmonic depth-of-field extends from 1.6 to $13.7 \mathrm{~cm}$. Thus, superharmonic imaging features a slightly lower penetration depth than fundamental or second harmonic imaging. However, the limited depth-of-field of superharmonic imaging also causes it to be less sensitive to near-field artifacts and reverberations compared to fundamental and second harmonic imaging, which is particularly useful for echocardiography.

\section{Simulation remarks}

The simulations presented here assumed a homogeneous propagation medium and used the material properties reported for cardiac tissue. However, in the case of echocardiography a propagating sound wave encounters a mix of cardiac tissue and blood. Although the B/A values of blood (6.0) and cardiac tissue (5.8) are similar, the attenuation of blood $\left(\sim 0.14 \mathrm{~dB} \cdot \mathrm{cm}^{-1} \cdot \mathrm{MHz}^{-1.2}\right)$ is about $2-3$ times lower than that of cardiac tissue $\left(0.52 \mathrm{~dB} \cdot \mathrm{cm}^{-1} \cdot \mathrm{MHz}^{-1}\right){ }^{25}$ Therefore, the axial pulse-echo pressures reported here apply to a worst-case scenario.

The MI is defined as the derated peak negative pressure divided by the square root of the frequency in $\mathrm{MHz} .{ }^{37} \mathrm{How}-$ ever, in this article the peak negative pressure has not been derated for the calculation of the MI. If the derated peak negative pressure had been used for the MI calculation, the pulse-echo pressure at focus for fundamental and second harmonic imaging would increase by at least $\sim 16 \mathrm{~dB}$. However, the superharmonic is made up from the third to the fifth harmonics. The third harmonic component would also increase by at least $\sim 16 \mathrm{~dB}$, but the fourth and fifth harmonics would increase by more than $\sim 22$ and $\sim 28 \mathrm{~dB}$, respectively. Thus, the backscattered superharmonic pressure would effectively increase somewhat compared to the intensities obtained using the fundamental and second harmonic imaging modalities.

In these simulations the backscattered power in the direction of the receiving aperture is equal to the incident power. Thus, the frequency dependent character of the backscattering was ignored in this comparison. This hypothesis is valid for the fundamental of $3.5 \mathrm{MHz}$, second, and third harmonics of $3.6 \mathrm{MHz}$, as these frequencies are almost the same. However, taking into account the power frequency dependency of the backscattering in biological tissue, ${ }^{25}$ the backscattering is expected to be larger for the fourth and fifth harmonics. Hence, in this respect again a worst-case scenario has been considered with regard to the superharmonic. It is important to mention that the contribution of those 
harmonics to superharmonic imaging is much lower than the one of the third. Therefore, the approximation to assume a frequency independent character of the backscattering was deemed acceptable.

\section{CONCLUSIONS}

It is the increased roll-off in combination with the progressive build-up of the harmonic beams that leads to a reduced sensitivity to off-axis scatterers, and a better suppression of near-field artifacts and reverberation in harmonic images compared to a fundamental image, despite the increased beamwidths around focus. Therefore, superharmonic imaging is particularly suited for echocardiography, where the imaging window is limited by the ribs and there is often a focus on high contrast borders between cardiac tissue and blood. The reduced spreading of the superharmonic beam in combination with a reduced roll-off and a higher axial resolution should lead to images of higher resolution compared to those produced by fundamental and second harmonic imaging. The drawback of superharmonic imaging is its lower pulse-echo peak pressure compared to second harmonic imaging. This leads to either a slightly lower depth-offield or the requirement for a more sensitive transducer in cases where mainly tissue is imaged, such as in abdominal imaging. However, this drawback is reduced in practical echocardiographic situations, where the sound wave encounters blood next to tissue. In this case, the lower attenuation of blood, in combination with the approximately equal nonlinearity, actually makes that the ratios between the superharmonics and the second harmonic become more favorable for superharmonic imaging, as compared to the considered tissue-only case.

In summary, superharmonic imaging appears particularly suited for echocardiography and is expected to improve the image quality of this modality at the cost of slight reduction in depth-of-field.

\section{ACKNOWLEDGMENTS}

This research was supported by the Dutch Technology Foundation (STW). The authors like to thank Koen van Dongen from the Section Acoustical Imaging and Sound Control, Faculty of Applied Sciences, Delft University of Technology, for facilitating the INCS simulations.

${ }^{1}$ F. Tranquart, N. Grenier, V. Eder, and L. Pourcelot, "Clinical use of ultrasound tissue harmonic imaging," Ultrasound Med. Biol. 25, 889-894 (1999).

${ }^{2}$ M. Averkiou, D. Roundhill, and J. Powers, "A new imaging technique based on the nonlinear properties of tissues," in Proceedings of the IEEE Ultrason. Symp., Toronto, Canada (1997), pp. 1561-1566.

${ }^{3}$ B. Ward, A. Baker, and V. Humphrey, "Nonlinear propagation applied to the improvement of resolution in diagnostic medical ultrasound," J. Acoust. Soc. Am. 101, 143-154 (1997).

${ }^{4}$ J. Kasprzak, B. Paelinck, F. Ten Cate, W. Vletter, N. De Jong, D. Poldermans, A. Elhendy, A. Bouakaz, and J. Roelandt, "Comparison of native and contrast-enhanced harmonic echocardiography for visualization of left ventricular endocardial border," Am. J. Cardiol. 83, 211-217 (1999).

${ }^{5}$ J. Thomas and D. Rubin, "Tissue harmonic imaging: Why does it work?," J. Am. Soc. Echocardiogr. 11, 803-808 (1998).

${ }^{6}$ R. Shapiro, J. Wagreich, R. Parsons, A. Stancato-Pasik, H. Yeh, and R. Lao, "Tissue harmonic imaging sonography: evaluation of image quality compared with conventional sonography," AJR, Am. J. Roentgenol. 171, 1203-1206 (1998).

${ }^{7} \mathrm{~V}$. Humphrey, "Nonlinear propagation in ultrasonic fields: measurements modelling and harmonic imaging," Ultrasonics 38, 267-272 (2000).

${ }^{8}$ A. Bouakaz and N. de Jong, "Native tissue imaging at superharmonic frequencies," IEEE Trans. Ultrason. Ferroelectr. Freq. Control 50, 496-506 (2003).

${ }^{9}$ R. Kompfner and R. A. Lemons, "Nonlinear acoustic microscopy," Appl. Phys. Lett. 28, 295-297 (1976).

${ }^{10}$ T. Muir and E. Carstensen, "Prediction of nonlinear acoustic effects at biomedical frequencies and intensities," Ultrasound Med. Biol. 6, 345-357 (1980).

${ }^{11}$ H. Starritt, F. Duck, A. Hawkins, and V. Humphrey, "The development of harmonic dis- tortion in pulsed finite-amplitude ultrasound passing through liver," Phys. Med. Bio. 31, 1401-1409 (1986).

${ }^{12}$ G. Matte, P. van Neer, J. Huijssen, M. Verweij, and N. de Jong, "Parameter optimization of interleaved phased arrays for transthoracic and abdominal applications of super harmonic imaging," IEEE Trans. Ultrason. Ferroelectr. Freq. Control 58, 533-546 (2011).

${ }^{13}$ A. Bouakaz, S. Frigstad, F. ten Cate, and N. de Jong, "Super harmonic imaging: a new imaging technique for improved contrast detection," Ultrasound Med. Biol. 28, 59-68 (2002).

${ }^{14}$ Q. Ma, D. Zhang, X. Gong, and Y. Ma, "Investigation of superharmonic sound propagation and imaging in biological tissues in-vitro," J. Acoust. Soc. Am. 119, 2518-2523 (2006).

${ }^{15}$ P. van Neer, G. Matte, M. Danilouchkine, C. Prins, F. van den Adel, and N. de Jong, "Super harmonic imaging: development of an interleaved phased array transducer," IEEE Trans. Ultrason. Ferroelectr. Freq. Control 57, 455-468 (2010).

${ }^{16}$ R. Zemp, J. Tavakkoli, and R. Cobbold, "Modeling of nonlinear ultrasound propagation in tissue from array transducers," J. Acoust. Soc. Am. 113, 139-152 (2003).

${ }^{17}$ J. Huijssen, M. Verweij, and N. de Jong, "Greens function method for modeling nonlinear three-dimensional pulsed acoustic fields in diagnostic ultrasound including tissue-like attenuation," in Proceedings of the IEEE Ultrason. Symp., Beijing, China (2008), pp. 375-378.

${ }^{18}$ J. Huijssen, "Modeling of nonlinear medical diagnostic ultrasound," Ph.D. thesis, Delft University of Technology, 35-120 and 171-186 (2008).

${ }^{19} \mathrm{~J}$. Huijssen and M. Verweij, "An iterative method for the computation of nonlinear, wide-angle, pulsed acoustic fields of medical diagnostic transducers," J. Acoust. Soc. Am. 127, 33-44 (2010).

${ }^{20}$ E. Zabolotskaya and R. Khokhlov, "Quasi-plane waves in the nonlinear acoustics of confined beams," Sovi. Phys. Acoust. 15, 3540 (1969).

${ }^{21}$ V. Kuznetsov, "Equations of nonlinear acoustics," Sov. Phys. Acoust. 16, 467-470 (1971).

${ }^{22}$ M. Hamilton and C. Morfey, "Model equations," in Nonlinear Acoustics, edited by M. Hamilton and D. Blackstock (Academic, San Diego, 1998), pp. 41-63.

${ }^{23}$ S. Aanonsen, J. Barkve, J. Naze Tjøtta, S. Tjøtta, “Distortion and harmonic generation in the nearfield of a finite amplitude sound beam," J. Acoust. Soc. Am 74, 749-768 (1984).

${ }^{24}$ A. Pierce, Acoustics (Acoustical Society of America, Melville, NY, 1989), pp. 555-562.

${ }^{25}$ F. Duck, Physical Properties of Tissues (Academic, San Diego, 1990), pp. 99-123.

${ }^{26}$ T. Szabo, Diagnostic Ultrasound Imaging: Inside Out (Elsevier Academic, San Diego, 2004), pp. 73-75 and 535.

${ }^{27}$ L. Demi, K. van Dongen, and M. Verweij, “A contrast source method for nonlinear acoustic wave fields in media with spatially inhomogeneous attenuation," J. Acoust. Soc. Am. 129, 1221-1230 (2011).

${ }^{28}$ Y. Lee and M. Hamilton, "Time-domain modeling of pulsed finiteamplitude sound beams," J. Acoust. Soc. Am. 97, 906-917 (1995).

${ }^{29} \mathrm{X}$. Yang and R. Cleveland, "Time domain simulation of nonlinear acoustic beams generated by rectangular pistons with application to harmonic imaging," J. Acoust. Soc. Am. 117, 113-123 (2005).

${ }^{30}$ T. Szabo, "Time-domain wave-equations for lossy media obeying a frequency power-law,” J. Acoust. Soc. Am. 96, 491-500 (1994).

${ }^{31}$ M. Voormolen, "3d harmonic echocardiography," Ph.D. thesis, Erasmus University Rotterdam, (2007), pp. 11-31.

${ }^{32} \mathrm{~W}$. Ames, Numerical Methods for Partial Differential Equations (Academic, New York, 1992), pp. 208-210.

${ }^{33}$ R. Cleveland, M. Hamilton, and D. Blackstock, "Time-domain modeling of finite-amplitude sound in relaxing fluids," J. Acoust. Soc. Am. 99, 3312-3318 (1996). 
${ }^{34} \mathrm{M}$. Verweij and J. Huijssen, "A filtered convolution method for the computation of acoustic wave fields in very large spatiotemporal domains," J. Acoust. Soc. Am. 125, 1868-1878 (2009).

${ }^{35}$ G. Matte, P. van Neer, J. Borsboom, M. Verweij, and N. de Jong, "A new frequency compounding technique for super harmonic imaging," in Proceedings of the IEEE Ultrason. Symp., Beijing, China (2008), pp. 357-360.
${ }^{36}$ P. van Neer, M. Danilouchkine, G. Matte, M. Verweij, and N. de Jong, "Dual pulse frequency compounded super harmonic imaging for phased array transducers," in Proceedings of the IEEE Ultrason. Symp., Rome, Italy (2009), pp. 381-384.

${ }^{37}$ R. Cobbold, Foundations of Biomedical Ultrasound (Oxford University Press, New York, 2007), p. 516. 